\title{
Revisión de metodologías de arranque óptimo de generación para el restablecimiento de sistemas de potencia considerando fuentes de energía convencionales y renovables no convencionales*
}

\author{
Ricardo Andrés Pardo** \\ Jesús María López-Lezama***
}

\author{
Recibido: 28/05/2019 - Aceptado: 02/08/2019 \\ https://doi.org/10.22395/rium.v19n36a9
}

\begin{abstract}
Resumen
Después de un apagón a gran escala, el restablecimiento del sistema eléctrico de potencia (SEP) debe realizarse rápidamente. Para ello, se debe restablecer el parque de generación, el sistema de transmisión y finalmente, la demanda desatendida. Para obtener un proceso acelerado de restablecimiento es necesario establecer metodologías de arranque de unidades de generación, que pongan en marcha, primero, las unidades con arranque en negro y, después, se les lleve potencia de arranque a otras unidades de generación sin esta característica, mediante una ruta de transmisión factible. En este artículo se presenta una revisión de diferentes metodologías de arranque óptimo de generadores para el restablecimiento del SEP, reportadas en la literatura científica teniendo en cuenta la integración de fuentes de energía renovables no convencionales. Dentro de esta revisión se destaca que los métodos heurísticos, aunque son efectivos, no se utilizan en la operación de tiempo real por su alto costo computacional.
\end{abstract}

Palabras clave: generación; optimización; renovables no convencionales; restablecimiento; secuencia óptima; sistemas de potencia.

* Artículo asociado al proyecto de investigación titulado: Metodología de arranque óptimo de generación para el restablecimiento de sistemas de potencia considerando fuentes de energía convencionales y renovables no convencionales, el cual se encuentra en curso.

** Estudiante de Maestría en Ingeniería, Grupo de Investigación en Manejo Eficiente de la Energía (Gimel), Departamento de Ingeniería Eléctrica, Universidad de Antioquia. Medellín, Colombia. Correo electrónico: ricardo.pardo@udea.edu.co. Orcid: http://orcid.org/0000-0002-2575-7494

*** Profesor del Departamento de Ingeniería Eléctrica, Grupo de Investigación en Manejo Eficiente de la Energía (Gimel), Universidad de Antioquia. Medellín, Colombia. Correo electrónico: jmaria.lopez@udea.edu.co. Orcid: http://orcid.org/0000-0002-2369-6173 


\title{
A Literature Review Of Optimal Generation Start-Up Methodologies For Power System Restoration Considering Conventional And Non- Conventional Renewable Energy Sources
}

\begin{abstract}
After a large-scale blackout power system restoration must be accomplished as soon as possible. For this, the generation must be initially restored, then, the transmission system, and finally the load pick up must be completed. To obtain a faster restoration process, it is necessary to establish start-up methodologies for generating units that first start those units that provide black start, and then take the starting power to other generation units without this characteristic, by means of a feasible transmission route. This paper presents a review of different methodologies of optimal generation start-up for power system restoration reported in the scientific literature taking into account the integration of non-conventional renewable energy sources. Within this review it is highlighted that heuristic methods, despite of being effective, are not used in real-time operation due to their high computational cost.
\end{abstract}

Keywords: generation; optimization; optimal sequence; power system; renewable no conventional; restoring.

\section{Review of generation optimal start methodologies for the recovery of power systems considering conventional and renewable- unconventional power sources}

\begin{abstract}
Resumen
After a big scale shutdown, the recovery of the "Electric Power System" (EPS) must be done quickly. For that, the generation park and the transmission system must be restored, and, finally, the demand must be unattended. For getting an accelerated process of this restoring process it is necessary to establish start methodologies for generation units capable of first starting the units with a black start and then conducting start power to other generation units without this feature through a feasible transmission route. In this article, we present a revision of different optimal start methodologies for generators for the restoring of the EPS reported in the scientific literature and involving renewable and unconventional power sources integration. Within this review, we highlight that the heuristics methods, although effective, are not used in the real-time operation for their high computational cost.
\end{abstract}

Keywords: generation; optimization; unconventional renewables; restoring; optimal sequence; power systems. 


\section{INTRODUCCIÓN}

El desarrollo de las sociedades modernas depende en gran medida del suministro de energía eléctrica; por esto, cuando se presentan dificultades en la prestación del servicio se crean traumatismos que son difícilmente predecibles y cuantificables [1].

Los sistemas de potencia son diseñados y operados bajo principios de seguridad y confiabilidad. Sin embargo, construir y operar un sistema totalmente confiable no es económicamente viable. Esta confiabilidad parcial, hace que eventos indeseables de gran magnitud sobre el SEP puedan originar un apagón parcial o total, tal como se ha experimentado en años recientes con apagones en diferentes partes del mundo [2].

Restablecer cuando se tienen recursos de generación convencionales de energía hidráulica y térmica es un proceso complejo que involucra devolver al sistema a una operación normal después de una interrupción súbita. Este proceso involucra un gran número de restricciones asociadas a los recursos de generación, transmisión, distribución y demanda [3]. Los operadores del mercado se anticipan a esta clase de eventos estableciendo estrategias de restablecimiento para evaluar los posibles escenarios o condiciones del sistema ante un apagón.

Por otra parte, las Fuentes de Energía Renovables no Convencionales (Fernc) presentan una operación del sistema con generación no controlada y distribuida, disminución en la autonomía de las subestaciones e incremento de coordinación necesaria con diferentes actores del sistema [4]. Bajo esta perspectiva, el proceso de restablecimiento del SEP incorporando Fernc es mucho más compleja en la actualidad y cambia dinámicamente con la implementación de nuevas tecnologías [5]. Un modelo de optimización que involucre Fernc en el restablecimiento de generación debe introducir variables de decisión que permitan obtener soluciones robustas, para ajustarse a las condiciones cambiantes del sistema.

En este artículo se presenta una revisión de las diferentes metodologías utilizadas para solucionar el problema de restablecimiento, desde una perspectiva de optimización de procesos. Para ello se han consultado las bases de datos IEEE Explorer y Science Direct desde el año 2000 hasta el año 2019, realizando filtros de búsqueda referentes con metodologías de restablecimiento de sistemas de potencia con fuentes de generación convencionales y no convencionales.

La estructura del artículo es la siguiente: en la sección 1 se expone el problema de restablecimiento, donde se especifican los retos que tienen que asumir los operadores del SEP durante este proceso y los desafíos que se presentan con la introducción de Fernc en los SEP. En la sección 2 se realiza una descripción de las diferentes metodologías 
utilizadas para resolver el problema de restablecimiento mediante la optimización de arranque de unidades de generación, la construcción de un camino factible de transmisión y el restablecimiento de la carga en el menor tiempo posible. En esta sección se presenta un cuadro comparativo, en orden cronológico, de las metodologías revisadas, categorizándolas de acuerdo a los recursos de generación disponibles en el SEP. Finalmente, en la sección 3 se presentan las conclusiones encontradas respecto a las metodologías con mayor viabilidad y adaptabilidad en la operación del SEP.

\section{EL PROBLEMA DE RESTABLECIMIENTO}

Uno de los principales factores que hacen complicada la tarea de restablecimiento de un SEP es la pérdida de conciencia situacional que se puede dar al presentarse un escenario extremo en la operación de tiempo real; también la indisponibilidad de los principales recursos del SEP y la no familiaridad del personal operativo con las condiciones inusuales en un estado de apagón del sistema [6].

\subsection{Retos en un proceso de restablecimiento}

Un proceso de restablecimiento implica los esfuerzos coordinados de todos los agentes y debe ir orientado a cumplir los siguientes objetivos: 1) una evaluación rápida y precisa de las condiciones actuales del SEP; 2) un restablecimiento rápido pero seguro de los recursos de generación; 3) una reconfiguración adecuada del sistema de transmisión con criterios de confiabilidad y seguridad; y 4) el restablecimiento de cargas prioritarias y aquellas necesarias para estabilizar el sistema.

Cumplir estos objetivos durante el restablecimiento del SEP no es una tarea sencilla, debido a que después de una falla en el sistema se pueden presentan los siguientes inconvenientes que pueden llegar a ser críticos y no permitir que el sistema recobre una operación normal y estable [7]:

- Altos voltajes debido a la falta de generación para soporte de potencia reactiva

- Oscilaciones de frecuencia y grandes variaciones de carga

- Fallas en el arranque en negro de algunas plantas

- Cierres no exitosos debido a diferencias angulares significativas

- Retrasos en la identificación de la falla

- Retrasos por evaluación del estado de interruptores

- La total dependencia de la generación térmica es un factor restrictivo en la velocidad del restablecimiento 
- Problemas de comunicación y dificultades en la conmutación de algunos centros de control de transmisión y la falta de información en sitio.

La problemática descrita conlleva grandes dificultades a los operadores de red (OR) en el momento de ejecutar un restablecimiento en tiempo real. Por ejemplo, según establece la experiencia de algunos OR, durante el proceso de restablecimiento se pueden presentar topologías de red parciales que resultan inestables o inseguras, y provocan nuevamente la salida en cascada de los elementos de la red ya recuperados [8]. Esta situación que retrasa el proceso de restablecimiento, se debe a que se deja esta tarea en manos de la experticia de los OR y no de algoritmos que apoyen estos procedimientos. Es por esta razón que resulta de vital importancia tener la ayuda de una metodología sistémica que oriente a los ingenieros de las salas de control en tiempo real, a seleccionar el siguiente paso y evaluar posibles nuevos puntos de operación durante el proceso de restablecimiento.

El desarrollo de metodologías para el restablecimiento de un SEP proporciona recomendaciones para llevar al sistema a un punto de operación seguro y confiable mediante la verificación de las variables de control que lo conforman. Adicionalmente, este tipo de metodologías reduce la incertidumbre que se genera al normalizar un SEP, en cuanto al comportamiento de sus componentes que pueden afectar nuevamente el proceso de toma de carga.

\subsection{Etapas del proceso de restablecimiento}

Una aproximación común para simplificar esta tarea es dividir el proceso de restablecimiento por etapas: preparación del parque de generación, restablecimiento de la red de transmisión y atención de la demanda. El hilo conductor es la disponibilidad de generación en cada etapa del restablecimiento, de tal manera que se pueda ir restableciendo la demanda desatendida. De esta manera, con una capacidad de generación disponible es posible atender la demanda del SEP y mantener en todo momento el balance generación-demanda necesario para estabilizar el sistema de energía y devolverlo a su condición normal.

\subsubsection{Restablecimiento con generación convencional}

La problemática que se presenta en el proceso de restablecimiento de recursos de generación radica en las características propias de las máquinas, las cuales deben ser consideradas en la sincronización de las unidades a la red eléctrica. Este hecho hace que el proceso de rearranque de unidades no sea tan simple e intuitivo como se espera. Por ejemplo, se debe tener en cuenta que las plantas de generación se clasifican en unidades de arranque en negro (BS por sus siglas en inglés), y unidades sin arranque 
en negro (NBS por sus siglas en inglés). Las unidades BS son limitadas en el SEP y pueden arrancar autónomamente sin necesidad de una fuente de alimentación externa; por otro lado, las unidades NBS requieren tensión a través de una fuente externa para volverlas a poner en marcha [9]. Adicionalmente, se debe tener en cuenta que los recursos de generación poseen inflexibilidades debido a sus características técnicas como tiempos mínimos de arranque y parada de los recursos, los cambios de potencia permitidos entre periodos y los mínimos técnicos de las máquinas, entre otras características [10].

Debido al número limitado generadores con arranque en negro y las diferentes restricciones para el arranque de unidades de generación, el problema radica en determinar la máxima disponibilidad de generación encontrando la secuencia óptima de arranque de todas las unidades de generación en el sistema. Como resultado es importante que durante el restablecimiento del sistema, sea maximizada la capacidad disponible de generación en el menor tiempo posible.

\subsubsection{Restablecimiento de un SEP con participación de Fernc}

Con la creciente participación de las Fernc en los SEP, los OR enfrentan retos adicionales en los restablecimientos. A diferencia de las fuentes convencionales de generación que permiten determinar con cierta certeza el valor de MW generados en cada instante de tiempo, las Fernc presentan una gran componente de incertidumbre, siendo la eólica y solar fotovoltaica (PV) las más comunes actualmente en los SEP [11], [12].

A medida que los parques eólicos se han masificado en los SEP, el riesgo de apagones es mayor debido a la fluctuación e incertidumbre de la energía eólica [13]. Los parques eólicos a gran escala deben participar en la restauración de los SEP [14], [15]. El principal inconveniente radica en la disponibilidad de potencia cuando sea necesaria, pues esta es función de la velocidad del viento que es de naturaleza aleatoria.

Por otro lado, la dependencia de la radiación solar implica cambios importantes en las componentes de generación; el hecho más marcado se presenta en las horas del atardecer donde la demanda eléctrica comienza a buscar su máximo valor del día, mientras que el sol se oculta y con ello se produce una reducción importante de la generación solar, hasta hacerse nula [16]. El sistema debe estar preparado no solo para responder al aumento de la demanda, sino además a la pérdida de generación solar lo que produce un doble efecto en las necesidades de incrementar la generación [17].

Los principales desafíos al proceso de restablecimiento introducidos por las Fernc se presentan a continuación [18]: 
- La salida de generación de Fernc es altamente fluctuante y puede modificar el valor y dirección de la cargabilidad de la red. Las unidades BS necesitan operar siempre en un rango dado de potencia activa.

- La generación de renovables opera autónomamente sin posibilidad de control remoto. El único control es la desconexión del punto de conexión donde ellas están conectadas, donde también están conectados otros clientes.

- Las fuentes de generación renovable son susceptibles a cambios súbitos de la frecuencia. Por lo tanto, cualquier desviación de frecuencia desencadena la conexión o la desconexión de la mayoría de las instalaciones fotovoltaicas. El posterior impacto de tensión y frecuencia de tal fenómeno podría llevar al apagón de islas recién restablecidas.

\section{METODOLOGÍAS PARA EL RESTABLECIMIENTO DE SEP}

Con la implementación de Fernc al SEP, las metodologías de restablecimiento con participación netamente de fuentes convencionales de energía han tenido que ajustarse a los desafíos tecnológicos de las fuentes no convencionales. A continuación, se describen las principales metodologías reportadas en las bases de datos IEEE Explorer y Science Direct desde el año 2000 hasta el año 2019, realizando filtros de búsqueda referentes con metodologías de restablecimiento del SEP utilizando fuentes convencionales y no convencionales de energía.

\subsection{Metodologías de restablecimiento con fuentes convencionales de energía}

En la fase de reconfiguración de la red eléctrica, la capacidad de generación obtenida después del arranque en negro de las unidades es utilizada para restablecer otras unidades importantes en el sistema y subestaciones en una secuencia razonable. Asimismo, se debe establecer una red troncal estable y los cimientos para el restablecimiento completo de la demanda [19]. Debido a estas características complejas la reconfiguración de la red necesita ser llevada a cabo bajo la guía de una estrategia de restablecimiento.

Una estrategia de restablecimiento de tres estados es propuesta en [20] para gestionar este proceso; por ejemplo, restablecer en primera instancia las unidades de generación principales, y después las subestaciones más importantes para luego restablecer la interconexión regional. Para lograr este tipo de estrategias se proponen investigaciones relacionadas con la optimización de la secuencia de arranque de unidades, la determinación de la red troncal o el esqueleto del restablecimiento, la optimización de la ruta de restauración y el restablecimiento en paralelo de la red de transmisión [21]. 
Un problema de restablecimiento puede ser formulado como un problema de optimización con restricciones no lineales de carácter multi-objetivo y multi-estado [22]. Algunas estrategias de planes de restablecimiento para asistir a los operadores en la toma de decisiones durante el proceso de restablecimiento han sido desarrolladas y aplicadas en [23]. Métodos heurísticos como los propuestos en [24] y [25] han sido utilizados para solucionar este problema de optimización combinatoria, pero el tiempo computacional es tan grande que se vuelve impráctico aplicarlo en un proceso de restablecimiento real. Enfoques novedosos que imitan las decisiones tomadas por los OR como algoritmos genéticos [26], redes neurales artificiales [27], lógica difusa [28] y redes de Petri [29] han sido desarrollados. Sin embargo, una de las debilidades de estos métodos radica en su poca aplicabilidad en la operación de tiempo real. Esto debido a que requieren del ajuste de varios parámetros lo que implica un costo computacional alto comparado con la celeridad necesaria en el proceso de restablecimiento.

Algunas herramientas de optimización convencional también han sido propuestas para mejorar la exactitud de las soluciones. Programación matemática [22], programación dinámica [30], indicadores de restablecimiento [31], [32], técnicas de programación entera-mixta [33], son algunas de las herramientas utilizadas para abordar el problema de restablecimiento.

Las investigaciones relacionadas con la secuencia de arranque óptimo pueden ser divididas en dos categorías: métodos de toma de decisiones y métodos de optimización múltiple. En [34] se desarrolla un modelo de toma de decisiones para determinar la secuencia de arranque de acuerdo a ciertas reglas o políticas establecidas. Una serie de estrategias de restablecimiento son aplicadas para optimizar la secuencia de arranque de unidades con el objetivo de maximizar la capacidad de generación potencial a restablecer de acuerdo a la potencia de arranque, tiempo de arranque en caliente, la rampa de entrada y el intervalo de tiempo máximo crítico de las unidades de generación.

Un gran número de estudios establece la secuencia de arranque de unidades como un problema de optimización y emplea programación no lineal y métodos de inteligencia artificial para solucionar este problema. En [33] se formula la secuencia de arranque de generación como un problema de programación lineal entero mixto, utilizando técnicas de transformación sobre las curvas de capacidad de generación las cuales son no lineales. En [35], la secuencia de arranque óptima es tratada como un proceso de restablecimiento de dos capas, denominadas unidades de restablecimiento de capas de trabajo y de planta. En esta propuesta, se utiliza el método de optimización lexicográfica para solucionar el problema de optimización multiobjetivo resultante.

Un modelo de optimización multi-objetivo para la secuencia de arranque de unidades es propuesto en [36], estableciendo una estrategia de particionamiento del sistema 
y buscando tiempos de respuesta de restablecimiento cortos. El algoritmo NSGA-II es aplicado para encontrar el frente óptimo de Pareto, mientras que el algoritmo de Dijkstra es utilizado para encontrar caminos de restablecimiento en la identificación de la secuencia de restablecimiento de unidades.

Debido a que los métodos de dos fases tienen como resultado una baja calidad de solución, en [37] se establece un modelo de optimización multi-objetivo mejorado de una fase donde la metodología implementada establece ponderaciones primarias y secundarias en objetivos de optimización. Esta metodología se desarrolla tomando simultáneamente la optimización de la secuencia de restablecimiento de las unidades de generación y la reconfiguración de la red. Por otra parte, en [38] se aborda el problema de restablecimiento de arranque de unidades de generación mediante optimización binivel. En este caso el nivel superior determina la secuencia de arranque óptima de unidades NBS mediante el uso de algoritmos de optimización basados en enseñanzaaprendizaje, y en el nivel inferior determina el camino de transmisión óptima con un mínimo número de movimientos de interruptores y máxima confiabilidad entre dos nodos utilizando teoría de grafos.

\subsection{Metodologías de restablecimiento implementando Fernc}

Como se observa hasta el momento, el común denominador en el desarrollo de las estrategias de restablecimiento, es utilizar los recursos de generación convencionales como un problema de primer nivel mediante el uso inicial de unidades hidráulicas y térmicas; para seguir con un proceso de segundo nivel con el restablecimiento de caminos de transmisión y carga, los cuales deben tener cierto margen de estabilidad. No obstante, en [39] se establece que el futuro de las estrategias de restablecimiento deben alinearse con el cambio de paradigma de la red. Esto se debe a la fuerte penetración a los SEP actuales de recursos Fernc acoplados con el incremento de esquemas de corriente directa (DC).

\subsubsection{Metodologías de restablecimiento con Fernc de tipo eólico}

El reciente desarrollo en el control de frecuencia de parques eólicos ha mejorado la integración de esta energía en el sistema eléctrico. Pese a ello, el riesgo de apagones también se ha incrementado debido a la volatilidad e incertidumbre de la generación eólica. Se han reportado pocas investigaciones sobre la función de restauración potencial de los parques eólicos en los sistemas de energía [21]. Dado que el tiempo de arranque de los aerogeneradores es más corto que las unidades NBS, algunos parques eólicos tienen la función potencial de servir como unidades BS bajo ciertas circunstancias. El algoritmo de optimización luciérnaga es usado en [40] para encontrar la secuencia 
óptima de restablecimiento de NBS, el camino óptimo de transmisión y la secuencia de restablecimiento óptima de carga con la integración de parques eólicos en el sistema.

En [37] se presenta un modelo de optimización de secuencia óptima que tiene en cuenta una unidad híbrida de arranque en negro que contiene una turbina de gas de combustión, un parque eólico y un compensador estático síncrono mediante un modelo de control de tres niveles. Esta metodología plantea un esquema de control que permite a los aerogeneradores de velocidad variable participar efectivamente en la regulación de voltaje y frecuencia del sistema. Cuando los parques eólicos con un almacenamiento de energía pueden guiarse por una estrategia de control adecuada, son de gran ayuda para impulsar el restablecimiento del sistema. Por otro lado, en [41] se enfocan en mejorar el proceso de restablecimiento para adaptar la alta participación de parques eólicos y tomar todas las ventajas de este tipo de generación durante el proceso de restablecimiento. En este artículo se discute la propiedad estocástica de la generación eólica, y se desarrolla un método de programación dinámica para que la generación eólica contribuya racionalmente en el proceso de restablecer el SEP.

\subsubsection{Metodologías de restablecimiento con Fernc de tipo fotovoltaica - PV}

En comparación con las investigaciones realizadas para las estrategias de arranque en generadores eólicos; el estudio de estrategias de arranque con generación fotovoltaica - $\mathrm{PV}$ - se ha realizado de manera diferente. El hecho anteriormente mencionado se debe a la excelente habilidad de autoarranque que poseen las unidades de generación PV y que pueden considerarse como unidades BS [42]. La influencia de este tipo de generación dentro del SEP ha empezado a ser estudiada en redes con configuración radial, microrredes y GD; las cuales pueden quedar aisladas del sistema ante eventuales fallas. Por ejemplo, en [43] se investiga el problema del diseño el tamaño, y la localización óptima de baterías de almacenamiento y generación PV para mejorar la resiliencia de los SEP debido a la propiedad de auto arranque de las unidades.

En [44] se plantea una estrategia de control para una microrred AC aislada con un sistema de almacenamiento híbrido, incluyendo baterías y supercapacitores. Dependiendo del estado de carga de las baterías, los sistemas PV pueden trabajar de acuerdo a las características técnicas de carga de las unidades de almacenamiento. Estos elementos son útiles en el proceso de restablecimiento debido a que su característica de almacenamiento hace que se puedan modelar como unidades BS, y proporcionar al SEP una alternativa temporal de atención de demanda prioritaria y alimentación de recursos NBS.

Dentro del problema de arranque de generadores para restablecimiento, en [45] se considera la diferencia entre unidades PV y generadores BS convencionales, y se 
propone una estrategia de restablecimiento de la red en dos fases. En la fase 1, se selecciona una unidad térmica mediante el algoritmo de Floyd, la cual se arranca mediante una unidad PV con el objetivo de maximizar la capacidad de arranque del sistema; mientras en la fase 2 la unidad arrancada en la fase 1 es utilizada como fuente principal de potencia en tanto la unidad PV se utiliza como una fuente auxiliar para realizar el restablecimiento.

\subsubsection{Metodologías de restablecimiento con Fernc en microrredes y GD}

La aparición de Fernc incorporadas en los sistemas de potencia, mejora la capacidad de recuperación automática y permite que los sistemas de distribución se recuperen más rápidamente en caso de un apagón. Una microrred puede operar en modo aislado en el momento que ocurra un apagón. En [46] se plantea una estrategia de restablecimiento basado en teoría de grafos incorporando microrredes. Esta estrategia maximiza la carga restablecida y minimiza el número de movimientos empleados por el operador de red. Los algoritmos de búsqueda de árbol de expansión se aplican para encontrar las estrategias de restauración candidatas modelando las microrredes como alimentadores ficticios.

Un enfoque bidireccional simultáneo en [47] investiga secuencias de restablecimiento de arranque en negro. Para ello establece las estrategias de control que deben ser implementadas por una microrred con unidades BS en su interior y la posterior operación en red aislada. En [48] se trata el problema de programación de recursos de generación multietapa en un restablecimiento, en una microrred aislada con múltiple generación distribuida (GD) y configuración radial. Dos métodos estocásticos son usados para solucionar este problema. Sobre condiciones adecuadas, GD puede mantener una cierta carga atendida en una microrred después de un apagón, dar algún soporte de potencia a microrredes adjuntas, o incluso a la red principal si hay GD sincronizada correctamente [5].

Además, en [49] se propone un sistema descentralizado multiagente para el proceso de restablecimiento con microrredes y GD. En este método cada agente es asociado con un consumidor o micro generador que se comunica con otro para lograr una decisión común de restablecimiento. Mediante una analogía del problema binario de la mochila (binary knapsack problem), los agentes encuentran la mejor secuencia de conexión de carga durante el restablecimiento de la red.

En la tabla 1 se presenta una breve síntesis de la clasificación de las metodologías de restablecimiento en orden cronológico, y de acuerdo al tipo de generación empleada. Se puede observar que hasta el año 2012 los estudios fueron enfocados al restablecimiento de SEP con fuentes de generación convencionales (hidráulica, térmica y nuclear). Pos- 
teriormente y debido al problema ambiental que se empezó a evidenciar a nivel mundial con el calentamiento global, diferentes naciones del mundo a través del protocolo de Kyoto se comprometieron a reducir las emisiones de gases de efecto invernadero [50]. Una de las medidas tomadas fue fomentar la introducción de generación renovable no convencional (predominantemente eólica y solar), lo cual hizo necesario involucrar en el análisis de restablecimiento de SEP la implementación de Fernc.

Estos análisis debieron realizarse debido a que este nuevo tipo de tecnologías presentan un comportamiento diferente al de fuentes convencionales: la alta variabilidad y dependencia del recurso primario, la conexión a la red al utilizar componentes DC y su efecto en el comportamiento dinámico que estos elementos tienen en el sistema.

Estas razones hicieron que las investigaciones se inclinaran hacia el estudio de modelos de varios niveles debido a la complejidad del problema, al utilizar Fernc tanto en la red de transmisión como en la red de distribución (DER), y el efecto que tienen en la construcción de la topología ante apagones parciales o totales de la red.

Tabla 1. Clasificación de las metodologías de restablecimiento de acuerdo al tipo de tecnología de generación aplicada organizadas cronológicamente

\begin{tabular}{clccc}
\hline $\begin{array}{c}\text { Año } \\
\text { publicación }\end{array}$ & \multicolumn{1}{c}{ Metodología } & Referencia $\begin{array}{c}\text { Unidades } \\
\text { conven- } \\
\text { cionales }\end{array}$ & $\begin{array}{c}\text { Microrredes } \\
\text { convencionales }\end{array}$ & $\begin{array}{c}\text { Integración } \\
\text { total de } \\
\text { recursos }\end{array}$ \\
\hline 2000 & Redes de Petri & {$[29]$} & $\mathrm{X}$ \\
\hline & Lógica difusa & {$[28]$} & $\mathrm{X}$ \\
\hline 2002 & AG, búsqueda tabú & {$[25]$} & $\mathrm{X}$ \\
\hline 2003 & Redes neuronales & {$[27]$} & $\mathrm{X}$ \\
\hline 2004 & Teoría de grafos y árboles de decisión & {$[24]$} & $\mathrm{X}$ \\
\hline 2005 & AG mutado & {$[26]$} & $\mathrm{X}$ \\
\hline 2008 & Programación dinámica & {$[30]$} & $\mathrm{X}$ \\
\hline 2010 & Indicadores de restablecimiento PTDF & {$[31]$} & $\mathrm{X}$ & \\
\hline & Pareto-Dijkstra NSGA-II & {$[36]$} & $\mathrm{X}$ & \\
\hline 2011 & LMIP Binivel & {$[33]$} & $\mathrm{X}$ & \\
\hline 2012 & Optimización lexicográfica & {$[35]$} & $\mathrm{X}$ & \\
\hline 2014 & Árboles de expansión en microrredes & {$[46]$} & & $\mathrm{X}$ \\
\hline & Teoría de grafos ponderada & {$[37]$} & & $\mathrm{X}$ \\
\hline
\end{tabular}




\begin{tabular}{|c|c|c|c|c|c|}
\hline $\begin{array}{c}\text { Año } \\
\text { publicación }\end{array}$ & Metodología & Referencia & $\begin{array}{c}\text { Unidades } \\
\text { conven- } \\
\text { cionales }\end{array}$ & $\begin{array}{l}\text { Microrredes } \\
\text { y recursos no } \\
\text { convencionales }\end{array}$ & $\begin{array}{l}\text { Integración } \\
\text { total de } \\
\text { recursos }\end{array}$ \\
\hline \multirow{3}{*}{2015} & Localización optima de generación PV & [43] & & $\mathrm{X}$ & \\
\hline & Algoritmos luciérnaga & {$[40]$} & & & $\mathrm{X}$ \\
\hline & $\begin{array}{l}\text { Modelamiento de unidades BS en } \\
\text { microrredes aisladas }\end{array}$ & [48] & & $\mathrm{X}$ & \\
\hline \multirow[t]{2}{*}{2016} & Estrategias de control de unidades BS & [47] & & $\mathrm{X}$ & \\
\hline & $\begin{array}{l}\text { Programación dinámica con } \\
\text { incertidumbre }\end{array}$ & [41] & & & $\mathrm{X}$ \\
\hline 2017 & Optimización jerárquica & {$[45]$} & & $\mathrm{X}$ & \\
\hline 2018 & $\begin{array}{l}\text { Técnicas de optimización } \\
\text { enseñanza-aprendizaje }\end{array}$ & {$[51]$} & & & $\mathrm{X}$ \\
\hline \multirow{2}{*}{2019} & Localización óptima de generación PV & [43] & & $\mathrm{X}$ & \\
\hline & NLMIP multiobjetivo & [44] & & $\mathrm{X}$ & \\
\hline
\end{tabular}

Fuente: elaboración propia

\section{CONCLUSIONES}

Las metodologías que abordan la problemática de la secuencia de arranque óptimo de recursos de generación, representan una herramienta esencial para resolver el problema de restablecimiento ante apagones totales y parciales del SEP. Mediante este tipo de herramientas se busca dar directrices a los operadores, de tal manera que se pueda agilizar el proceso haciéndolo más metódico, seguro y confiable.

Con base en la revisión bibliográfica realizada, se puede evidenciar que hasta el año 2007 las metodologías heurísticas con unidades convencionales fueron el foco de investigación. Posteriormente, desde el año 2007 hasta el año 2012 se tuvo una fuerte tendencia a realizar investigaciones con programación matemática debido a la eficiencia computacional, y la posibilidad de encontrar un óptimo global del problema de optimización abordado.

Desde el año 2014 hasta la fecha y debido a la progresiva participación de las Fernc en los SEP, las investigaciones han ido encaminadas a estudiar esta creciente tendencia. La naturaleza intermitente de las Fernc y la incertidumbre en la duración del viento o el sol como recursos primarios de generación, plantean nuevos desafíos en la tarea clásica de programación de arranque de unidades de generación para el restablecimiento de los SEP. Por otra parte, el modelado de estos recursos constituye 
una tarea de vital importancia en el desarrollo de metodologías que implementen estas tecnologías. Esto servirá como herramienta en la especificación de requerimientos mínimos establecidos en las políticas energéticas, como parámetro esencial para la conexión de estos recursos a la red, los cuales deben ir alineados con los criterios de economía, seguridad y confiabilidad de los SEP.

Finalmente, se evidencia que son pocos los trabajos que incorporan integralmente las tecnologías convencionales y no convencionales de forma simultánea en el problema de restablecimiento, por lo que se puede deducir que es un buen nicho de investigación y es pertinente el estudio de este tema debido a la creciente implementación de las tecnologías no convencionales en los SEP.

\section{REFERENCIAS}

[1] Y.-L. Yuan-Kang, "Literature Review of Power System Blackouts", Energy Procedia, vol. 141, pp. 428-431, 2017. DOI: 10.1007/s40565-016-0219-2

[2] O. P. Veloza y F. Santamaría, "Analysis of major blackouts from 2003 to 2015 : Classi fi cation of incidents and review of main causes", Electr. J., vol. 29, n. ${ }^{\circ}$ 7, pp. 42--9, 2016. DOI: 10.1016/j.tej.2016.08.006

[3] R. F. Chu y A. T. Holen, "Generation Capability Dispatch for Bulk Power System Restoration ", IEEE Trans. Power Syst., vol. 8, n. ${ }^{\circ}$ 1, pp. 316-325, 1993. DOI: 10.1109/59.221225

[4] Y. Nakanishi, T. Saito y R. Yokoyama, "Wind power generation”, Energy Technol. Roadmaps Japan Futur. Energy Syst. Based Feasible Technol. Beyond 2030, pp. 307-322, 2016. DOI: 10.1007/978-4-431-55951-1_19

[5] L. Che, M. Khodayar y M. Shahidehpour, "Only connect: Microgrids for distribution system restoration”, IEEE Power Energy Mag., vol. 12, n. ${ }^{\circ}$ 1, pp. 70-81, 2014. DOI: 10.1109/ MPE.2013.2286317

[6] M. Socha, "Metodología técnico-pedagógica para el entrenamiento de operadores en la tarea de restablecimiento de la operación del sistema de potencia", Universidad Nacional de Colombia, 2010. 10.1016/S0142-0615(00)00061-2

[7] D. Lindenmeyer, H. W. Dommel y M. M. Adibi, "Power system restoration - a bibliographical survey", Int. J. Electr. Power Energy Syst., vol. 23, n. 3, pp. 219-227, 2001. 10.1016/ S0142-0615(00)00061-2

[8] XM, “Despacho de generación”, 2018. [Internet]. Disponible en: https://www.xm.com.co/ Paginas/Generacion/despacho.aspx.

[9] W. Sun, S. Member, C. Liu, S. Liu y A. B. S. Resources, "Black Start Capability Assessment in Power System Restoration”, 2011 IEEE Power Energy Soc. Gen. Meet., pp. 1-7, 2011. 10.1109/PES.2011.6039752

[10] C. Mario y C. Posada, "Modelo de optimización para las plantas térmicas de generación de ciclo combinado en el despacho económico", Universidad Nacional de Colombia, 2009. 
[11] T. A. Ogden Araya, "Métodos de identificación y predicción de rampas en sistemas eléctricos con generación intermitente”, p. 100, 2015.

[12] A. M. El-zonkoly, "Renewable energy sources for complete optimal power system black-start restoration”, IET Gener. Transm. Distrib., vol. 9, n. ${ }^{\circ}$ 6, pp. 531-539, 2015. DOI: 10.1049/ iet-gtd.2014.0646

[13] A. El-Zonkoy, "Electrical Power and Energy Systems Power system single step restoration incorporating cold load pickup aided by distributed generation", Int. J. Electr. Power Energy Syst., vol. 35, n. ${ }^{\circ}$ 1, pp. 186-193, 2012. DOI: 10.1016/j.ijepes.2011.10.012

[14] V. Quaschning, "Wind Power Systems - Electricity from Thin Air”, en Renewable Energy and Climate Change, 1a ed., L. John Wiley \& Sons, Ed. Berlin, Germany: Wiley-IEEE Press, pp. 165-190, 2011. DOI: 10.1002/9781119994381.ch8

[15] L. Seca, H. Costa, C. L. Moreira y J. A. P. Lopes, "An innovative strategy for power system restoration using utility scale wind parks", en Proceedings of IREP Symposium: Bulk Power System Dynamics and Control - IX Optimization, Security and Control of the Emerging Power Grid, IREP 2013, pp. 1-8, 2013. DOI: 10.1109/IREP.2013.6629404

[16] G. M. Masters, "Chapter 7 The Solar Resource", en Renewable and Efficient Electric Power Systems, pp. 385-443, 2004. DOI: 10.1002/0471668826

[17] E. Vinson, A. Jurado y P. Gigli, "Estudio de un sistema de distribución con alta penetración de generación de energía solar", 2015.[Internet]. Disponibe en https://www.editores-srl.com. ar/sites/default/files/ie296_electrotecnica_estudio_de_un_sistema_de_distribucion.pdf

[18] J. Sprooten, T. Gunst, C. Mestdag y O. Bronckart, "Power system restoration with high penetration level of renewable generation - New challenges and strategies", 2014 Saudi Arab. Smart Grid Conf., pp. 1-8, 2014. DOI: 10.1109/SASG.2014.7274287

[19] C. Shen, P. Kaufmann y M. Braun, “Optimizing the generator start-up sequence after a power system blackout”, IEEE Power Energy Soc. Gen. Meet., vol. 2014-Octob, núm. October, pp. 1-5, 2014. DOI: 10.1109/PESGM.2014.6938799

[20] C. Shen, P. Kaufmann, C. Hachmann y M. Braun, "Three-stage power system restoration methodology considering renewable energies", Int. J. Electr. Power Energy Syst., vol. 94, pp. 287-299, 2018. DOI: 10.1016/j.ijepes.2017.07.007

[21] Y. Liu, R. Fan y V. Terzija, "Power system restoration: a literature review from 2006 to 2016", J. Mod. Power Syst. Clean Energy, vol. 4, n. 3 3, pp. 332-341, 2016. DOI: 10.1007/ s40565-016-0219-2

[22] T. Nagata, S. Hatakeyama, M. Yasouka, y H. Sasaki, "An efficient method for power distribution system restoration based on mathematical programming and operation strategy", Power Syst. Technol. 2000. Proceedings. PowerCon 2000. Int. Conf., vol. 3, pp. 1545-1550, 2000. DOI: 10.1109/ICPST.2000.898201

[23] R. Urrea y J. Gómez, "Modelo experto para apoyo en restablecimiento de sistemas de potencia”, en XIII Eriac, Cigre, pp. 1-9, 2009. 
[24] T. N. S. V. S. M. R. Sudhakar, "Heuristic based strategy for the restoration problem in electric power distribution systems", en International Conference on Power System Technology POWERCON 2004, n. ${ }^{\circ}$ 1, pp. 21-24, 2004. DOI: 10.1109/ICPST.2004.1460071

[25] S. Toune, H. Fudo, T. Genji Y. Fukuyama, y Y. Nakanishi, "Comparative study of modern heuristic algorithms to service restoration in distribution systems", IEEE Trans. Power Deliv., vol. 17 , n. ${ }^{\circ}$, pp. 173-181, 2002. DOI: 10.1109/61.974205

[26] K. Prasad, R. Ranjan, N. C. Sahoo y a Chaturvedi, "Optimal reconfiguration of radial distribution systems using a fuzzy mutated genetic algorithm", IEEE Trans. Power Deliv., vol. 20 , n. $^{\circ} 2$, pp. 1211-1213, 2005. DOI: 10.1109/TPWRD.2005.844245

[27] A. S. Bretas y A. G. Phadke, "Artificial neural networks in power system restoration", IEEE Trans. Power Deliv., vol. 18, n. ${ }^{\circ}$ 4, pp. 1181-1186, 2003. DOI: 10.1109/TPWRD.2003.817500

[28] Y. Hsiao, C. Chien y S. Member, "Enhancement of Restoration Service in Distribution Systems Using a Combination Fuzzy - GA Method", Power, vol. 15, n. ${ }^{4}$ 4, pp. 1394-1400, 2000. DOI: $10.1109 / 59.898118$

[29] J. S. Wu, C. C. Liu, K. L. Liout y R. F. Chu, "A petri net algorithm for scheduling of generic restoration actions", Power Syst. Restor. Methodol. Implement. Strateg., vol. 12, n. ${ }^{\circ}$, pp. 538-545, 2000. DOI: 10.1109/9780470545607.ch71

[30] R. Pérez-Guerrero, G. T. Heydt, N. J. Jack, B. K. Keel y A. R. Castelhano, “Optimal Restoration of Distribution Systems Using Dynamic Programming”, IEEE Trans. Power Deliv., vol. 23, n. ${ }^{\circ}$ 3, pp. 1589-1596, 2008. DOI: 10.1109/TPWRD.2007.916112

[31] C. Wang, V. Vittal, V. S. Kolluri y S. Mandal, "PTDF-based automatic restoration path selection”, IEEE Trans. Power Syst., vol. 25, n. ${ }^{\circ}$, pp. 1686-1695, 2010. DOI: 10.1109/ TPWRS.2009.2037820

[32] M. P. Zuluaga Pareja, "Propuesta metodológica de tiempo real para el proceso de restablecimiento de un área operativa de un sistema eléctrico de potencia", Universidad Nacional de Colombia, 2017.

[33] W. Sun, C. C. Liu, y L. Zhang, "Optimal generator start-up strategy for bulk power system restoration”, IEEE Trans. Power Syst., vol. 26, n. 3, pp. 1357-1366, 2011. DOI: 10.1109/ TPWRS.2010.2089646

[34] F. Wen, G. Ledwich, C. Zhang, Z. Lin y Y. Xue, “Two-stage power network reconfiguration strategy considering node importance and restored generation capacity”, IET Gener. Transm. Distrib., vol. 8, n. ${ }^{\circ}$ 1, pp. 91-103, 2014. DOI: 10.1049/iet-gtd.2013.0065

[35] X. G. H. Zhong, "Optimisation of network reconfiguration based on a two-layer unit-restarting framework for power system restoration”, n. ${ }^{\circ}$ ag. 2011, pp. 693-700, 2012. DOI: 10.1049/ iet-gtd.2011.0591

[36] H. Wang, C. He y Y. Liu, "Pareto optimization of power system reconstruction using NSGA-II algorithm”, Asia-Pacific Power Energy Eng. Conf. APPEEC, n. ․ 1, pp. 1-5, 2010. DOI: 10.1109/ APPEEC.2010.5448326 
[37] W. Teng, H. Wang y Y. Jia, "Construction and control strategy research of black start unit containing wind farm”, IEEE Reg. 10 Annu. Int. Conf. Proceedings/TENCON, vol. 2016-Janua, n. ${ }^{\circ}$ 51177092, 2016. DOI: 10.1109/TENCON.2015.7372862

[38] A. Ketabi, A. Karimizadeh y M. Shahidehpour, "Optimal generation units start-up sequence during restoration of power system considering network reliability using bilevel optimization", Int. J. Electr. Power Energy Syst., vol. 104, n. 1, pp. 772-783, 2019. DOI: 10.1016/j.ijepes.2018.07.045

[39] D. K. Maina y N.-K. C. Nair, "Recent advancements on Power System Restoration”, 2017 IEEE Innov. Smart Grid Technol. - Asia, pp. 1-5, 2017. DOI: 10.1109/ISGT-Asia.2017.8378461

[40] A. El-Zonkoy, "Integration of wind power for optimal power system black-start restoration", Turkish J. Electr. Eng. Comput. Sci., vol. 23, pp. 1853-1866, 2015. DOI: 10.3906/elk-1402-267

[41] R. Hu, W. Hu, P. Li, C. Su y Z. Chen, "A Dynamic Programming based Method for Optimizing Power System Restoration with High Wind Power Penetration”, 2016 IEEE 8th Int. Power Electron. Motion Control Conf. (IPEMC-ECCE Asia), pp. 2022-2027, 2017. DOI: 10.1109/ IPEMC.2016.7512606

[42] A. E. B. Abu-elanien, M. M. A. Salama y K. B. Shaban, "Modern network reconfiguration techniques for service restoration in distribution systems : A step to a smarter grid", Alexandria Eng. J., vol. 57, n. ${ }^{\circ}$ 4, pp. 3959-3967, 2018. DOI: 10.1016/j.aej.2018.03.011

[43] B. Zhang, S. Member, P. Dehghanian, y S. Member, "Optimal Allocation of PV Generation and Battery Storage for Enhanced Resilience", IEEE Trans. Smart Grid, vol. 10, n. ${ }^{\circ}$ 1, pp. 535-545, 2019. DOI: 10.1109/TSG.2017.2747136

[44] S. Wen, "Energy Management and Coordinated Control Strategy of PV / HESS AC Microgrid During Islanded Operation”, IEEE Access, vol. 7, pp. 4432-4441, 2019. DOI: 10.1109/ ACCESS.2018.2887114

[45] Z. Jiang, F. Xiao, Q. Ai, Q. He y Q. Sun, “Two-phase integrated optimisation strategy for network restoration with photovoltaic generation”, J. Eng., vol. 2017, n. ${ }^{\circ} 13$, pp. 1076-1081, 2017. DOI: $10.1049 /$ joe.2017.0495

[46] J. Li, X. Y. Ma, C. C. Liu, y K. P. Schneider, "Distribution system restoration with microgrids using spanning tree search”, IEEE Trans. Power Syst., vol. 29, n. ${ }^{\circ}$ 6, pp. 3021-3029, 2014. DOI: 10.1109/TPWRS.2014.2312424

[47] C. L. Moreira, F. O. Resende y J. A. P. Lopes, "Using low voltage MicroGrids for service restoration”, IEEE Trans. Power Syst., vol. 22, n. ${ }^{\circ}$ 1, pp. 395-403, 2007. DOI: 10.1109/ TPWRS.2006.888989

[48] B. Zhao, X. Dong y J. Bornemann, "Service Restoration for a Renewable-Powered Microgrid in Unscheduled Island Mode", IEEE Trans. Smart Grid, vol. 6, n. ${ }^{\circ}$, pp. 1128-1136, 2015. DOI: $10.1109 /$ TSG.2014.2373272 
[49] J. P. P. Carvalho, M. Shafie-khah y G. Osório, "Multi-Agent System for Renewable Based Microgrid Restoration”, en 2018 International Conference on Smart Energy Systems and Technologies (SEST), pp. 1-6, 2018. DOI: 10.1109/SEST.2018.8495894

[50] N. Unidas, "Protocolo de Kyoto de la convención marco de las Naciones Unidas sobre el cambio climático", Kioto, Japán, 1998.

[51] H. Zhong y X. Gu, "Determination of optimal unit start-up sequences based on fuzzy AHP in power system restoration", 2011 4th Int. Conf. Electr. Util. Deregul. Restruct. Power Technol., pp. 1541-1545, 2011. DOI: 10.1109/DRPT.2011.5994141 